УДК 517.5

\author{
M. M. Sheremeta, M. S. Dobushovskyy
}

\title{
ON THE TWO-MEMBER ASYMPTOTIC OF YOUNG CONJUGATED FUNCTIONS
}

\begin{abstract}
M. M. Sheremeta, M. S. Dobushovskyy. On the two-member asymptotic of Young conjugated functions, Mat. Stud. 46 (2016), 178-188.

Let $P:(0 ;+\infty) \rightarrow+\infty$ with $p \not \equiv-\infty$. A connection between behavior of $P$ and the growth of the function $Q(\sigma)=\sup \{P(t)+\sigma t: t \geq 0\}$ is in the term of the two-member generalized asymptotic.
\end{abstract}

1. Introduction. Let $P$ be an arbitrary function different from $+\infty$ (it can achieve the value $-\infty$ but $P \neq \equiv-\infty)$ and let $Q(\sigma)=\sup \{P(t)+t \sigma: t \geq 0\}$. The functions $P$ and $Q$ are said to be Young conjugated functions. If we put $A=\sup \{\sigma: Q(\sigma)<+\infty\}$ then

$$
A=-\varlimsup_{t \rightarrow+\infty} P(t) / t
$$

Indeed, if $\sigma<A$ then $P(t)+t, \sigma<Q(\sigma)$ and, thus, $P(t) / t<-\sigma+Q(\sigma) / t$ for all $t \geq 0$, whence

$$
\varlimsup_{t \rightarrow+\infty} P(t) / t<-\sigma
$$

that is in view of the arbitrariness of $\sigma$ we have

$$
\varlimsup_{t \rightarrow+\infty} P(t) / t \leq-A .
$$

On the other hand, if $\sigma>A$ then $Q(\sigma)=+\infty$ and, therefore, for every $K \in(0,+\infty)$ there exists a sequence $\left(t_{k}\right) \uparrow+\infty$ such that $P\left(t_{k}\right)+\sigma t_{k} \geq K$, that is $P\left(t_{k}\right) / t_{k} \geq-\sigma+o(1)$ as $k \rightarrow \infty$, whence $\varlimsup_{t \rightarrow+\infty} P(t) / t \geq-A$.

Suppose that $-\infty<A \leq+\infty$ and by $\Omega(A)$ we denote the class of positive unbounded on $(-\infty, A)$ functions $\Phi$ such that the derivative $\Phi^{\prime}$ is positive continuously differentiable and increasing to $+\infty$ on $(-\infty, A)$. From now on, we denote by $\varphi$ the inverse function to $\Phi^{\prime}$, and let $\Psi(x)=x-\Phi(x) / \Phi^{\prime}(x)$ be the function associated with $\Phi$ in the sense of Newton. It is clear that the function $\varphi$ is continuously differentiable and increasing to $A$ on $(0,+\infty)$. The function $\Psi$ is $[1$, p. $30 ; 2-3]$ continuously differentiable and increasing to $A$ on $(-\infty, A)$. The following lemmas are proved in [3] and [1, p. 30-45].

Lemma 1. Let $A \in(-\infty,+\infty]$ and $\Phi \in \Omega(A)$. In order that $Q(\sigma) \leq \Phi(\sigma)$ for all $\sigma \in\left[\sigma_{0}, A\right)$, it is necessary and sufficient that $\ln P(t) \leq-t \Psi(\varphi(t))$ for all $t \geq t_{0}$.

2010 Mathematics Subject Classification: 33B50, 44A10.

Keywords: two-member asymptotic; Young conjugated functions.

doi:10.15330/ms.46.2.178-188

(C) M. M. Sheremeta, M. S. Dobushovskyy, 2016 
Lemma 2. Let $A \in(-\infty,+\infty]$ and $\Phi \in \Omega(A)$. If $P\left(t_{k}\right) \geq-t_{k} \Psi\left(\varphi\left(t_{k}\right)\right)$ for some sequence $\left(t_{k}\right)$ of positive numbers increasing to $+\infty$ then for all $k \geq k_{0}$ and all $\sigma \in\left[\varphi\left(t_{k}\right), \varphi\left(t_{k+1}\right)\right]$

$$
Q(\sigma) \geq \Phi(\sigma)+G_{1}\left(t_{k}, t_{k+1}, \Phi\right)-G_{2}\left(t_{k}, t_{k+1}, \Phi\right)
$$

where $([1, p .34 ; 4])$

$$
G_{1}(a, b, \Phi)=: \frac{a b}{b-a} \int_{a}^{b} \frac{\Phi(\varphi(q t))}{t^{2}} d t<G_{2}(a, b, \Phi)=: \Phi\left(\frac{1}{b-a} \int_{a}^{b} \varphi(q t) d t\right)
$$

for $0<a<b<+\infty$.

Lemma 3. Let $A \in(-\infty,+\infty], \Phi_{*} \in \Omega(A), \Phi^{*} \in \Omega(A)$ and $\Phi_{*}(\sigma) \leq Q(\sigma) \leq \Phi^{*}(\sigma)$ for all $\sigma \in\left(\sigma_{0}, A\right)$. Then $P(t) \leq-t \Psi^{*}\left(\varphi^{*}(t)\right)$ for all $t>t_{0}$ and there exists an increasing to $+\infty$ sequence $\left(t_{k}\right)$ of positive numbers such that $P\left(t_{k}\right) \geq-t_{k} \Psi_{*}\left(\varphi_{*}\left(t_{k}\right)\right)$ and

$$
G_{1}\left(t_{k}, t_{k+1}, \Phi^{*}\right) \geq \Phi_{1}\left(\varkappa_{k}\left(\Phi^{*}\right)\right), \quad \varkappa_{k}(\Phi)=\frac{1}{t_{k+1}-t_{k}} \int_{t_{k}}^{t_{k+1}} \varphi(t) d t
$$

where by $\Psi_{*}, \Psi^{*}, \varphi_{*}$ and $\varphi^{*}$ we denote the functions which correspond to $\Phi_{*}$ and $\Phi^{*}$.

Using the lemmas, in [5] it is found conditions on $P$ under which for example, $Q$ has two-member exponential asymptotic

$$
Q(\sigma)=T e^{\rho \sigma}+(1+o(1)) \tau e^{\rho \sigma} \quad(\sigma \rightarrow+\infty), \quad T>0,0<\rho_{1}<\rho<+\infty, \tau \in \mathbb{R},
$$

$Q$ has two-member exponential asymptotics

$$
Q(\sigma)=T \sigma^{p}+(1+o(1)) \tau \sigma^{p_{1}} \quad(\sigma \rightarrow+\infty), \quad T>0, p>1,0<p_{1}<p, \tau \in \mathbb{R},
$$

or

$$
Q(\sigma)=\frac{T}{|\sigma|^{p}}+\frac{(1+o(1)) \tau}{|\sigma|^{p_{1}}} \quad(\sigma \uparrow 0), \quad T>0,0<p_{1}<p<+\infty, \tau \in \mathbb{R} .
$$

For $A=+\infty$ a general two-member asymptotic of $Q$ is studied in [6-7].

Let $L^{0}$ be the class of positive continuously differentiable on $(0,+\infty)$ functions $l$ such that $x l^{\prime}(x)=O(l(x))$ as $x \rightarrow+\infty$. We remark that if $l \in L^{0}$ then $l((1+o(1)) x)=(1+o(1)) l(x)$ as $x \rightarrow+\infty$.

As in [6] we will say that a positive twice continuously differentiable increasing to $+\infty$ on $(-\infty,+\infty)$ function $\Phi_{2}$ is subordinated to $\Phi_{1} \in \Omega(+\infty)$ if $\Phi_{2}^{\prime \prime}(\sigma)=o\left(\Phi_{1}^{\prime \prime}(\sigma)\right), \Phi_{2}^{\prime}(\sigma)=$ $o\left(\sigma \Phi_{1}^{\prime \prime}(\sigma)\right)$ as $\sigma \rightarrow+\infty$ and $\Phi_{2}^{\prime}\left(\varphi_{1}\right) \in L^{0}$.

Theorem A $([6])$. Let $\Phi_{1} \in \Omega(+\infty), \varphi_{1}^{\prime} \in L^{0}, \Phi_{2}$ be subordinated to $\Phi_{1}$ and $\tau \in \mathbb{R}$. In order that

$$
Q(\sigma) \leq \Phi_{1}(\sigma)+(1+o(1)) \tau \Phi_{1}(\sigma), \quad \sigma \rightarrow+\infty
$$

it is necessary and sufficient that

$$
P(t) \leq-t \Psi_{1}\left(\varphi_{1}(t)\right)+(1+o(1)) \tau \Phi_{2}\left(\varphi_{1}(t)\right), \quad t \rightarrow+\infty .
$$


Theorem B ([6]). Let $\Phi_{1} \in \Omega(+\infty), \varphi_{1}^{\prime} \in L^{0}, \Phi_{2}$ be subordinated to $\Phi_{1}, \tau \in \mathbb{R}$ and

$$
\Phi_{j}^{\prime}\left(\sigma+O\left(\Phi_{2}^{\prime}(\sigma) / \Phi_{1}^{\prime \prime}(\sigma)\right)\right)=(1+o(1)) \Phi_{j}^{\prime}(\sigma) \quad(\sigma \rightarrow+\infty), \quad j=1,2 .
$$

If

$$
P\left(t_{n}\right) \geq-t_{n} \Psi_{1}\left(\varphi_{1}\left(t_{n}\right)\right)+(1+o(1)) \tau_{n} \Phi_{2}\left(\varphi_{1}\left(t_{n}\right)\right), \quad n \rightarrow+\infty,
$$

for a some increasing to $+\infty$ sequence $\left(t_{n}\right)$ such that

$$
t_{n+1}=(1+o(1)) t_{n}, \quad n \rightarrow \infty,
$$

and

$$
G_{2}\left(t_{n}, t_{n+1}, \Phi_{1}\right)-G_{1}\left(t_{n}, t_{n+1}, \Phi_{1}\right)=o\left(\Phi_{2}\left(\varphi_{1}\left(t_{n}\right)\right)\right), \quad n \rightarrow \infty
$$

then

$$
Q(\sigma) \geq \Phi_{1}(\sigma)+(1+o(1)) \tau \Phi_{1}(\sigma), \quad \sigma \rightarrow+\infty .
$$

Theorem C ([7]). Let $\Phi_{1} \in \Omega(+\infty), \varphi_{1}^{\prime} \in L^{0}, \Phi_{2}$ be subordinated to $\Phi_{1}, \tau \in \mathbb{R}$, the conditions (3) hold and (5) imply (6). Put

$$
\kappa_{n}\left(\Phi_{1}\right)=\frac{1}{t_{n+1}-t_{n}} \int_{t_{n}}^{t_{n+1}} \varphi_{1}(t) d t, \xi_{n}=\frac{\Phi_{2}\left(\varphi_{1}\left(t_{n+1}\right)\right)-\Phi_{2}\left(\varphi_{1}\left(t_{n+1}\right)\right)}{t_{n+1}-t_{n}}
$$

and suppose that

$$
\xi_{n} \Phi_{1}^{\prime}\left(\kappa_{n}\left(\Phi_{1}^{\prime}\right)+O\left(\xi_{n}\right)\right)=o\left(G_{2}\left(t_{n}, t_{n+1}, \Phi_{1}\right)\right), \quad n \rightarrow \infty
$$

and

$$
\Phi_{2}\left(\kappa_{n}\left(\Phi_{1}^{\prime}\right)+O\left(\xi_{n}\right)\right)=o\left(G_{2}\left(t_{n}, t_{n+1}, \Phi_{1}\right)\right), \quad n \rightarrow \infty
$$

In order that

$$
Q(\sigma)=\Phi_{1}(\sigma)+(1+o(1)) \tau \Phi_{1}(\sigma), \quad \sigma \rightarrow+\infty,
$$

it is necessary and sufficient that for every $\varepsilon>0$ :

1) for all $t \geq t_{0}=t_{0}(\varepsilon)$

$$
P(t) \leq-t \Psi_{1}\left(\varphi_{1}(t)\right)+(\tau+\varepsilon) \Phi_{2}\left(\varphi_{1}(t)\right)
$$

2) there exists an increasing to $+\infty$ sequence $\left(t_{n}\right)$ such that

$$
\left.P\left(t_{n}\right) \geq-t_{n} \Psi_{1}\left(\varphi_{1}\left(t_{n}\right)\right)+(\tau-\varepsilon)\right) \Phi_{2}\left(\varphi_{1}\left(t_{n}\right)\right), \quad n \rightarrow+\infty,
$$

and (6) holds.

In view of Theorem $\mathrm{C}$ the following problems arise ([7]).

1. For which function $\Phi_{1} \in \Omega(+\infty)$ and subordinated to $\Phi_{1}$ function $\Phi_{2}$ do relations (9) and (10) hold for every sequence $\left(t_{n}\right) \uparrow+\infty$ ?

2. For which functions $\Phi_{1} \in \Omega(+\infty)$ and $\Phi_{2}$ does relation (5) imply (6)?

Here we will discuss the set of forth problems and will generalize Theorems 1-3 in the case of any $A \in(-\infty,+\infty]$. Obviously, the general case $A \in(-\infty,+\infty)$ can be reduced to the case $A=0$ with $\sigma-A$ replacing $\sigma$.

2. Discussion of problems. The answer to the first problem is contained in the following proposition. 
Proposition 1. Let either $A=0$ or $A=+\infty, \Phi_{1} \in \Omega(A)$ and $\Phi_{2}$ be a positive twice continuously differentiable function on $(-\infty, A)$ increasing to $+\infty$. Suppose that $\Phi_{2}^{\prime}(\sigma)=$ $o\left(\sigma \Phi_{1}^{\prime \prime}(\sigma)\right), \sigma \Phi_{1}^{\prime}((1+o(1)) \sigma)=O\left(\Phi_{1}(\sigma)\right)$ and $\Phi_{2}((1+o(1)) \sigma)=o\left(\Phi_{1}(\sigma)\right)$ as $\sigma \uparrow A$. Then the relations (9) and (10) hold for every sequence $\left(t_{n}\right) \uparrow+\infty$.

Proof. The condition $\Phi_{2}^{\prime}(\sigma)=o\left(\sigma \Phi_{1}^{\prime \prime}(\sigma)\right)$ as $\sigma \uparrow A$ implies the relation $\Phi_{2}^{\prime}\left(\varphi_{1}(t)\right)=o\left(\varphi_{1}(t) \times\right.$ $\left.\times \Phi_{1}^{\prime \prime}\left(\varphi_{1}(t)\right)\right)$ as $t \rightarrow+\infty$. But $\Phi_{1}^{\prime \prime}\left(\varphi_{1}(t)\right)=1 / \varphi_{1}^{\prime}(t)$. Therefore, $\Phi_{2}^{\prime}(\varphi(t)) \varphi^{\prime}(t)=o(\varphi(t))$ as $t \rightarrow+\infty$ and, thus, in view of (8)

$$
\xi_{n}=\frac{\Phi_{2}\left(\varphi_{1}\left(t_{n+1}\right)\right)-\Phi_{2}\left(\varphi_{1}\left(t_{n}\right)\right)}{t_{n+1}-t_{n}}=\frac{1}{t_{n+1}-t_{n}} \int_{t_{n}}^{t_{n+1}} \Phi_{2}^{\prime}\left(\varphi_{1}(t)\right) p_{1}^{\prime}(t) d t=o\left(\kappa_{n}\left(\Phi_{1}\right), \quad n \rightarrow \infty .\right.
$$

Since $G_{2}\left(t_{n}, t_{n+1}, \Phi_{1}\right)=\Phi_{1}\left(\kappa_{n}\left(\Phi_{1}\right)\right)$ and $\sigma \Phi_{1}^{\prime}((1+o(1)) \sigma)=O\left(\Phi_{1}(\sigma)\right)$ as $\sigma \uparrow A$ hence we obtain (9). From the condition $\Phi_{2}((1+o(1)) \sigma)=o\left(\Phi_{1}(\sigma)\right)$ as $\sigma \uparrow A$ the relation (10) follows.

The assumptions of Proposition 1 are satisfied if $\Phi_{1}$ and $\Phi_{2}$ are power functions, that is $\Phi_{1}(\sigma)=\sigma^{p}(p>1), \Phi_{2}(\sigma)=\sigma^{p_{1}}\left(0<p_{1}<p\right)$ by $A=+\infty$ and $\Phi_{1}(\sigma)=1 /|\sigma|^{p}(p>0)$, $\Phi_{2}(\sigma)=1 /|\sigma|^{p_{1}}\left(0<p_{1}<p\right)$ by $A=0$. The exponential functions $\Phi_{1}(\sigma)=\exp \{\rho \sigma\}(\rho>0)$, $\Phi_{2}(\sigma)=\exp \left\{\rho_{1} \sigma\right\}\left(0<\rho_{1}<\rho\right)$ dissatisfy these condition. However, the following statement holds.

Proposition 2. Let $A=+\infty, \Phi_{1} \in \Omega(A)$ and $\Phi_{2}$ be a positive continuously differentiable function on $(-\infty, A)$ increasing to $+\infty$. Suppose that $\Phi_{2}^{\prime}(\sigma)=o\left(\Phi_{1}^{\prime \prime}(\sigma)\right), \Phi_{1}^{\prime}(\sigma+o(1))=$ $O\left(\Phi_{1}(\sigma)\right)$ and $\Phi_{2}(\sigma+o(1))=O\left(\Phi_{1}(\sigma)\right)$ as $\sigma \uparrow A$. Then the relations (9) and (10) hold for every sequence $\left(t_{n}\right) \uparrow+\infty$.

Proof. The condition $\Phi_{2}^{\prime}(\sigma)=o\left(\Phi_{1}^{\prime \prime}(\sigma)\right)$ as $\sigma \uparrow A$ implies the relation $\Phi_{2}^{\prime}\left(\varphi_{1}(t)\right) \varphi_{1}^{\prime}(t) \rightarrow 0$ as $t \rightarrow+\infty$ and, thus, $\xi_{n} \rightarrow 0$ as $n \rightarrow \infty$. Therefore, from the condition $\Phi_{1}^{\prime}(\sigma+o(1))=O\left(\Phi_{1}(\sigma)\right)$ we obtain (9) and from the condition $\Phi_{2}(\sigma+o(1))=o\left(\Phi_{1}(\sigma)\right)$ as $\sigma \uparrow A$, we obtain (10).

Now we consider the second problem. Suppose that $\Phi_{2}(\sigma)=o\left(\Phi_{1}(\sigma)\right)$ as $\sigma \uparrow A$. Since $G_{2}\left(t_{n}, t_{n+1}, \Phi_{1}\right) \geq \Phi_{1}\left(\varphi_{1}\left(t_{n}\right)\right)$ we have $\Phi_{2}\left(\varphi_{1}\left(t_{n}\right)\right)=o\left(G_{2}\left(t_{n}, t_{n+1}, \Phi_{1}\right)\right)$ as $n \rightarrow \infty$ and, therefore, from (6) it follows that

$$
G_{2}\left(t_{n}, t_{n+1}, \Phi_{1}\right)=(1+o(1)) G_{1}\left(t_{n}, t_{n+1}, \Phi_{1}\right), \quad n \rightarrow \infty .
$$

By $\Omega^{*}(A)$ we denote the class of functions $\Phi \in \Omega(A)$ such that (14) implies (5). Then the following proposition is true.

Proposition 3. Let either $A=0$ or $A=+\infty, \Phi_{1} \in \Omega^{*}(A)$, and $\Phi_{2}$ be a positive continuous function on $(-\infty, A)$ increasing to $+\infty$ such that $\Phi_{2}(\sigma)=o\left(\Phi_{1}(\sigma)\right)$ as $\sigma \uparrow A$. Then (6) implies (5).

The following problem arises: for which functions $\Phi_{1} \in \Omega(A)$ does relation (14) implies (5)?

We did not succeed to distinguish the general classes of functions for what (14) implies (5). However the class $\Omega^{*}(A)$ is nonempty. For example the following functionals belongs to this classes: an exponential function $\Phi(\sigma)=T e^{\rho \sigma}$ and a power function $\Phi(\sigma)=T \sigma^{p}$ for $\sigma \geq \sigma_{0}$ 
if $A=+\infty$ and a power function $\Phi(\sigma)=T|\sigma|^{-\rho}$ if $A=0$, where $T>0, \rho>0$ and $p>1$. We will show it when $T=\rho=1$ and $p=2$.

If $\Phi(\sigma)=e^{\sigma}$ then

$$
G_{1}\left(t_{n}, t_{n+1}, \Phi\right)=\frac{t_{n} t_{n+1}}{t_{n+1}-t_{n}} \ln \frac{t_{n+1}}{t_{n}}, \quad G_{2}\left(t_{n}, t_{n+1}, \Phi\right)=\exp \left\{\frac{t_{n+1} \ln t_{n+1}-t_{n} \ln t_{n}}{t_{n+1}-t_{n}}-1\right\} .
$$

Therefore, if we put $t_{n+1}=\left(1+\theta_{n}\right) t_{n}$ then

$$
G_{1}\left(t_{n}, t_{n+1}, \Phi\right)=t_{n} \frac{\left(1+\theta_{n}\right) \ln \left(1+\theta_{n}\right)}{\theta_{n}}, G_{2}\left(t_{n}, t_{n+1}, \Phi\right)=\frac{t_{n}}{e} \exp \left\{\frac{\left(1+\theta_{n}\right) \ln \left(1+\theta_{n}\right)}{\theta_{n}}\right\}
$$

and, thus,

$$
G_{n}=: \frac{G_{1}\left(t_{n}, t_{n+1}, \Phi\right)}{G_{2}\left(t_{n}, t_{n+1}, \Phi\right)}=e \eta_{n} e^{-\eta_{n}}, \quad \eta_{n}=: \frac{\left(1+\theta_{n}\right) \ln \left(1+\theta_{n}\right)}{\theta_{n}} .
$$

If there exists a sequence $\left(\theta_{n_{j}}\right), \theta_{n_{j}} \rightarrow+\infty$ then $\eta_{n_{j}} \rightarrow+\infty$ and $G_{n_{j}} \rightarrow 0$ as $j \rightarrow \infty$. If $\theta_{n_{j}} \rightarrow \theta \in(0,+\infty)$ then $\eta_{n_{j}} \rightarrow \eta=: \frac{(1+\theta) \ln (1+\theta)}{\theta}>0$ and $G_{n_{j}} \rightarrow e \eta e^{-\eta}<1$ as $j \rightarrow \infty$. Therefore, from (14) it follows that $\theta_{n} \rightarrow 0$ as $n \rightarrow \infty$ and, thus, (5) holds.

Now, let $\Phi(\sigma)=\sigma^{2}$ for $\sigma \geq \sigma_{0}$ and $t_{n+1}=\left(1+\theta_{n}\right) t_{n}$. Then $G_{1}\left(t_{n}, t_{n+1}, \Phi\right)=t_{n} t_{n+1} / 4=$ $=t_{n}\left(1+\theta_{n}\right) / 4, G_{2}\left(t_{n}, t_{n+1}, \Phi\right)=\left(t_{n}+t_{n+1}\right)^{2} / 16=t_{n}^{2}\left(2+\theta_{n}\right)^{2} / 16$ and, thus, $G_{n}=4(1+$ $\left.\theta_{n}\right) /\left(2+\theta_{n}\right)^{2}$, whence, as above, in view of (14) $\theta_{n} \rightarrow 0$ as $n \rightarrow \infty$ and, thus, (5) holds.

Finally, let $\Phi(\sigma)=1 /|\sigma|$. Then

$$
\begin{gathered}
G_{1}\left(t_{n}, t_{n+1}, \Phi\right)=\frac{2 \sqrt{t_{n} t_{n+1}}}{\sqrt{t_{n}}+\sqrt{t_{n+1}}}=\frac{2 \sqrt{t_{n}} \sqrt{1+\theta_{n}}}{\sqrt{1+\theta_{n}}+1} \\
G_{2}\left(t_{n}, t_{n+1}, \Phi\right)=\left(\sqrt{t_{n}}+\sqrt{t_{n+1}}\right) / 2=\sqrt{t_{n}}\left(\sqrt{1+\theta_{n}}+1\right) / 2
\end{gathered}
$$

and $G_{n}=4 \sqrt{1+\theta_{n}} /\left(\sqrt{1+\theta_{n}}+1\right)^{2}$, whence, as above, in view of (14) $\theta_{n} \rightarrow 0$ as $n \rightarrow \infty$ and, thus, (5) holds.

3. Preliminary statements. Let $A \in(-\infty,+\infty]$ and $\Phi_{1} \in \Omega(A)$. We will say that a positive twice continuously differentiable increasing to $+\infty$ on $(-\infty, A)$ a function $\Phi_{2}$ is weakly subordinated to $\Phi_{1} \in \Omega(+\infty)$ if $\Phi_{2}^{\prime \prime}(\sigma)=o\left(\Phi_{1}^{\prime \prime}(\sigma)\right)$ as $\sigma \uparrow A$ and $\Phi_{2}^{\prime}\left(\varphi_{1}\right) \in L^{0}$. We remark that $\Phi_{2}^{\prime}\left(\varphi_{1}\right) \in L^{0}$ iff $\Phi_{2}^{\prime \prime}(\sigma) / \Phi_{2}^{\prime}(\sigma)=O\left(\Phi_{1}^{\prime \prime}(\sigma) / \Phi_{1}^{\prime}(\sigma)\right)$ as $\sigma \uparrow A$.

Let $\tau \in \mathbb{R} \backslash\{0\}$ and either $A=0$ or $A=+\infty$. Suppose that $\Phi_{1} \in \Omega(A), \varphi_{1}^{\prime} \in L^{0}$ and $\Phi_{2}$ is weakly subordinated to $\Phi_{1}$. Since $\Phi_{2}^{\prime \prime}(\sigma)=o\left(\Phi_{1}^{\prime \prime}(\sigma)\right)$ as $\sigma \uparrow A$, there exists a function $\Phi \in \Omega(A)$ such that

$$
\Phi(\sigma)=\Phi_{1}(\sigma)+\tau \Phi_{2}(\sigma), \quad \sigma \in\left[\sigma_{0}(\tau), A\right) .
$$

Lemma 4. For the function (15) the following asymptotic equalities are true

$$
\varphi(t)=\varphi_{1}(t)-(1+o(1)) \tau \Phi_{2}^{\prime}\left(\varphi_{1}(t)\right) \varphi_{1}^{\prime}(t), \quad t \rightarrow+\infty
$$

and

$$
t \Psi(\varphi(t))=t \Psi_{1}\left(\varphi_{1}(t)\right)-(1+o(1)) \tau \Phi_{2}\left(\varphi_{1}(t)\right), \quad t \rightarrow+\infty .
$$

Proof. Clearly, the inverse function $\varphi$ to $\Phi$ satisfies the equation

$$
\Phi^{\prime}(\sigma)+\tau+\Phi_{2}^{\prime}(\sigma)=t
$$


Since $\Phi_{2}^{\prime}(\sigma)=o\left(\Phi_{1}^{\prime}(\sigma)\right)$ as $\sigma \uparrow A$, we look for a solution of (18) of the form

$$
\varphi(t)=\varphi_{1}(t-\alpha(t)), \alpha(t)=o(t)(t \rightarrow+\infty) .
$$

Substituting (19) in (18) and taking into account the condition $\Phi_{2}^{\prime}\left(\varphi_{1}\right) \in L^{0}$, we obtain

$$
\alpha(t)=\tau+\Phi_{2}^{\prime}\left(\varphi_{1}(t-\alpha(t))\right)=\tau \Phi_{2}^{\prime}\left(\varphi_{1}((1+o(1)) t)\right)=(1+o(1)) \tau \Phi_{2}^{\prime}\left(\varphi_{1}(t)\right), \quad t \rightarrow+\infty .
$$

On the other hand, in view the condition $\varphi_{1}^{\prime} \in L^{0}$ for some $\eta=\eta(t) \in[t-\alpha(t), t]$ we have

$$
\varphi_{1}(t)-\varphi_{1}(t-\alpha(t))=\varphi_{1}^{\prime}(\eta) \alpha(t)=(1+o(1)) \varphi_{1}^{\prime}(t) \alpha(t), \quad t \rightarrow+\infty .
$$

Therefore, (19) and (20) imply (16).

Since $(t \Psi(\varphi(t)))^{\prime}=\varphi(t)$ from (16) it follows that

$t \Psi(\varphi(t))-t_{0} \Psi\left(\varphi\left(t_{0}\right)\right)=t \Psi_{1}\left(\varphi_{1}(t)\right)-t_{0} \Psi_{1}\left(\varphi_{1}\left(t_{0}\right)\right)-(\tau+\varepsilon)(1+o(1))\left(\Phi_{2}^{\prime}\left(\varphi_{1}(t)\right)-\Phi_{2}^{\prime}\left(\varphi_{1}\left(t_{0}\right)\right)\right)$

as $t \rightarrow+\infty$. Since $\varphi_{1}(t) \uparrow A$ as $t \rightarrow+\infty$ and $\Phi_{2}(\sigma) \uparrow+\infty$ as $\sigma \uparrow A$, one has $\Phi_{2}\left(\varphi_{1}(t)\right) \uparrow+\infty$ as $t \rightarrow+\infty$. Therefore, the last inequality implies (17).

Lemma 5. Let $\Phi_{1} \in \Omega(A), \varphi_{1}^{\prime} \in L^{0}$ and $\Phi_{2}$ be weakly subordinated to $\Phi_{1}$. Suppose that $\Phi_{2}^{\prime}(\sigma)=o\left(\sigma \Phi_{1}^{\prime \prime}(\sigma)\right)$ as $\sigma \uparrow A$, the conditions (3) as $\sigma \uparrow A$ and (5) hold. Then for the function (15) the following asymptotic equality is true

$$
G_{2}\left(t_{n}, t_{n+1}, \Phi\right)-G_{1}\left(t_{n}, t_{n+1}, \Phi\right)=G_{2}\left(t_{n}, t_{n+1}, \Phi_{1}\right)-G_{1}\left(t_{n}, t_{n+1}, \Phi_{1}\right)+o\left(\Phi_{2}\left(\varphi_{1}\left(t_{n}\right)\right)\right)
$$

as $n \rightarrow \infty$.

Proof. If we define $\kappa_{n}\left(\Phi_{1}\right)$ and $\xi_{n}$ as in (8) then from (16) we obtain

$$
\begin{gathered}
\kappa_{n}(\Phi)=\frac{1}{t_{n+1}-t_{n}} \int_{t_{n}}^{t_{n+1}} \varphi(t) d t=\frac{1}{t_{n+1}-t_{n}} \int_{t_{n}}^{t_{n+1}} \varphi_{1}(t) d t- \\
-\frac{(1+o(1)) \tau}{t_{n+1}-t_{n}} \int_{t_{n}}^{t_{n+1}} \tau \Phi_{2}^{\prime}\left(\varphi_{1}(t)\right) \varphi_{1}^{\prime}(t) d t=\kappa_{n}\left(\Phi_{1}\right)-(1+o(1)) \tau \xi_{n}, \quad n \rightarrow \infty .
\end{gathered}
$$

The condition $\Phi_{2}^{\prime}(\sigma)=o\left(\sigma \Phi_{1}^{\prime \prime}(\sigma)\right)$ as $\sigma \uparrow A$ implies the relation $\xi_{n}=o\left(\kappa_{n}\left(\Phi_{1}\right)\right)$ as $n \rightarrow \infty$. Therefore,

$$
\begin{gathered}
G_{2}\left(t_{n}, t_{n+1}, \Phi\right)=\Phi\left(\kappa_{n}(\Phi)\right)=\Phi_{1}\left(\kappa_{n}(\Phi)\right)+\tau \Phi_{2}\left(\kappa_{n}(\Phi)=\right. \\
=\Phi_{1}\left(\kappa_{n}\left(\Phi_{1}\right)-(1+o(1)) \tau \xi_{n}\right)+\tau \Phi_{2}\left(\kappa_{n}\left(\Phi_{1}\right)-(1+o(1)) \tau \xi_{n}\right), \quad n \rightarrow \infty .
\end{gathered}
$$

Since $\varphi_{1}^{\prime}((1+o(1)) x)=(1+o(1)) \varphi_{1}^{\prime}(x)$ as $x \rightarrow+\infty$ and $\Phi_{1}^{\prime \prime}\left(\varphi_{1}(x)\right)=1 / \varphi_{1}^{\prime}(x)$, we have $\Phi_{1}^{\prime \prime}\left(\varphi_{1}((1+o(1)) x)\right)=(1+o(1)) \Phi_{1}^{\prime \prime}\left(\varphi_{1}(x)\right)$ as $x \rightarrow+\infty$. The condition (5) implies the relation $\kappa_{n}\left(\Phi_{1}\right)=\varphi_{1}\left((1+o(1)) t_{n}\right)$ as $n \rightarrow \infty$. Therefore, in view of the condition $\Phi_{2}^{\prime}\left(\varphi_{1}\right) \in L^{0}$ we have

$$
\begin{gathered}
\frac{\Phi_{2}^{\prime}\left(\kappa_{n}\left(\Phi_{1}\right)\right.}{\Phi_{1}^{\prime \prime}\left(\kappa_{n}\left(\Phi_{1}\right)\right.}=\frac{\Phi_{2}^{\prime}\left(\varphi_{1}\left((1+o(1)) t_{n}\right)\right)}{\Phi_{1}^{\prime \prime}\left(\varphi_{1}\left((1+o(1)) t_{n}\right)\right)}=(1+o(1)) \frac{\Phi_{2}^{\prime}\left(\varphi_{1}\left(t_{n}\right)\right)}{\Phi_{1}^{\prime \prime}\left(\varphi_{1}\left(t_{n}\right)\right)}= \\
\quad=(1+o(1)) \Phi_{2}^{\prime}\left(\varphi_{1}\left(t_{n}\right)\right) \varphi_{1}^{\prime}\left(t_{n}\right)=(1+o(1)) \xi_{n}, \quad n \rightarrow \infty .
\end{gathered}
$$


Hence it follows that for some $\eta_{n} \in(0,1)$ by (3)

$$
\begin{gathered}
\Phi_{1}\left(\kappa_{n}\left(\Phi_{1}\right)-(1+o(1)) \tau \xi_{n}\right)-\Phi_{1}\left(\kappa_{n}\left(\Phi_{1}\right)\right)=-\Phi_{1}^{\prime}\left(\kappa_{n}\left(\Phi_{1}\right)-(1+o(1)) \eta_{n} \tau \xi_{n}\right)(1+o(1)) \tau \xi_{n}= \\
\quad=-(1+o(1)) \tau \xi_{n} \Phi_{1}^{\prime}\left(\kappa_{n}\left(\Phi_{1}\right)-(1+o(1)) \eta_{n} \tau \frac{\Phi_{2}^{\prime}\left(\kappa_{n}\left(\Phi_{1}\right)\right.}{\Phi_{1}^{\prime \prime}\left(\kappa_{n}\left(\Phi_{1}\right)\right.}\right)=-(1+o(1)) \tau \xi_{n} \Phi_{1}^{\prime}\left(\kappa_{n}\left(\Phi_{1}\right)\right)
\end{gathered}
$$

as $n \rightarrow \infty$, and by analogy

$$
\begin{gathered}
\Phi_{2}\left(\kappa_{n}\left(\Phi_{1}\right)-(1+o(1)) \tau \xi_{n}\right)-\Phi_{2}\left(\kappa_{n}\left(\Phi_{1}\right)\right)=-\Phi_{2}^{\prime}\left(\kappa_{n}\left(\Phi_{1}\right)-(1+o(1)) \eta_{n} \tau \xi_{n}\right)(1+o(1)) \tau \xi_{n}= \\
\quad=-(1+o(1)) \tau \xi_{n} \Phi_{2}^{\prime}\left(\kappa_{n}\left(\Phi_{1}\right)-(1+o(1)) \eta_{n} \tau \frac{\Phi_{2}^{\prime}\left(\kappa_{n}\left(\Phi_{1}\right)\right.}{\Phi_{1}^{\prime \prime}\left(\kappa_{n}\left(\Phi_{1}\right)\right.}\right)=-(1+o(1)) \tau \xi_{n} \Phi_{2}^{\prime}\left(\kappa_{n}\left(\Phi_{1}\right)\right)
\end{gathered}
$$

as $n \rightarrow \infty$. Thus,

$$
\begin{aligned}
& G_{2}\left(t_{n}, t_{n+1}, \Phi\right)=G_{2}\left(t_{n}, t_{n+1}, \Phi_{1}\right)+\tau \Phi_{2}\left(\kappa_{n}\left(\Phi_{1}\right)\right)- \\
& -(1+o(1)) \tau \xi_{n} \Phi_{1}^{\prime}\left(\kappa_{n}\left(\Phi_{1}\right)\right)-(1+o(1)) \tau \xi_{n} \Phi_{2}^{\prime}\left(\kappa_{n}\left(\Phi_{1}\right)\right), \quad n \rightarrow \infty .
\end{aligned}
$$

On the other hand, in view of (17) and the equality $\varphi(x)=\left(x \Psi(\varphi(x))^{\prime}\right.$ we have

$$
\begin{gathered}
G_{1}\left(t_{n}, t_{n+1}, \Phi\right)=\frac{t_{n} t_{n+1}}{t_{n+1}-t_{n}} \int_{t_{n}}^{t_{n+1}} \Phi(\varphi(t)) d\left(-\frac{1}{t}\right)=\frac{t_{n} t_{n+1}}{t_{n+1}-t_{n}}\left(\Psi\left(\varphi\left(t_{n+1}\right)\right)-\right. \\
\Psi\left(\varphi\left(t_{n}\right)\right)=t_{n}\left(\frac{t_{n+1} \Psi\left(\varphi\left(t_{n+1}\right)\right)-t_{n} \Psi\left(\varphi\left(t_{n}\right)\right)}{t_{n+1}-t_{n}}-\Psi\left(\varphi\left(t_{n}\right)\right)\right)= \\
=t_{n} \kappa_{n}(\Phi)-t_{n} \Psi\left(\varphi\left(t_{n}\right)\right)=t_{n} \kappa_{n}\left(\Phi_{1}\right)-(1+o(1)) \tau \xi_{n} t_{n}- \\
-t_{n} \Psi_{1}\left(\varphi_{1}\left(t_{n}\right)\right)+(1+o(1)) \tau \Phi_{2}\left(\varphi_{1}\left(t_{n}\right)\right)= \\
=G_{1}\left(t_{n}, t_{n+1}, \Phi_{1}\right)-(1+o(1)) \tau \xi_{n} t_{n}+(1+o(1)) \tau \Phi_{2}\left(\varphi_{1}\left(t_{n}\right)\right), \quad n \rightarrow \infty .
\end{gathered}
$$

From the conditions $\Phi_{2}^{\prime}\left(\varphi_{1}\right) \in L^{0}$ and $\varphi_{1}^{\prime} \in L^{0}$ it follows that

$$
\begin{gathered}
0<\varlimsup_{x \rightarrow+\infty} \frac{x \Phi_{2}^{\prime}\left(\varphi_{1}(x)\right) \varphi_{1}^{\prime}(x)}{\Phi_{2}\left(\varphi_{1}(x)\right)} \leq \\
\leq \varlimsup_{x \rightarrow+\infty} \frac{\Phi_{2}^{\prime}\left(\varphi_{1}(x)\right) \varphi_{1}^{\prime}(x)+x \Phi_{2}^{\prime \prime}\left(\varphi_{1}(x)\right) \varphi_{1}^{\prime}(x)^{2}+x \Phi_{2}^{\prime}\left(\varphi_{1}(x)\right) \varphi_{1}^{\prime \prime}(x)}{\Phi_{2}^{\prime}\left(\varphi_{1}(x)\right) \varphi_{1}^{\prime}(x)} \leq \\
\leq 1+\varlimsup_{x \rightarrow+\infty} \frac{x \Phi_{2}^{\prime \prime}\left(\varphi_{1}(x)\right) \varphi_{1}^{\prime}(x)}{\Phi_{2}^{\prime}\left(\varphi_{1}(x)\right)}+\varlimsup_{x \rightarrow+\infty} \frac{x \varphi_{1}^{\prime \prime}(x)}{\varphi_{1}^{\prime \prime}(x)}<+\infty,
\end{gathered}
$$

i. e. $\Phi_{2}\left(\varphi_{1}\right) \in L^{0}$. Therefore, taking into account (5) and $\Phi_{2}\left(\kappa_{n}\right)=(1+o(1)) \Phi_{2}\left(\varphi\left(t_{n}\right)\right)$ as $n \rightarrow \infty$, from (22) and (23) we obtain

$$
\begin{gathered}
G_{2}\left(t_{n}, t_{n+1}, \Phi\right)-G_{1}\left(t_{n}, t_{n+1}, \Phi\right)=G_{2}\left(t_{n}, t_{n+1}, \Phi_{1}\right)-G_{1}\left(t_{n}, t_{n+1}, \Phi_{1}\right)- \\
-(1+o(1)) \tau \xi_{n} \Phi_{1}^{\prime}\left(\kappa_{n}\left(\Phi_{1}\right)\right)-(1+o(1)) \tau \xi_{n} \Phi_{2}^{\prime}\left(\kappa_{n}\left(\Phi_{1}\right)\right)+(1+o(1)) \tau \xi_{n} t_{n}+ \\
+o\left(\Phi_{2}\left(\varphi_{1}\left(t_{n}\right)\right)\right), \quad n \rightarrow \infty .
\end{gathered}
$$

Since $\xi_{n}=\Phi_{2}^{\prime}\left(\varphi_{1}\left(\eta_{n}\right)\right) \varphi_{1}^{\prime}\left(\eta_{n}\right)\left(t_{n}<\eta_{n}<t_{n+1}\right), t_{n+1}=(1+o(1)) t_{n}$ as $n \rightarrow+\infty, \Phi_{2}^{\prime}\left(\varphi_{1}\right) \in L^{0}$ and $\varphi_{1}^{\prime} \in L^{0}$ we have

$$
\xi_{n}=(1+o(1)) \Phi_{2}^{\prime}\left(\varphi_{1}\left(t_{n}\right)\right) \varphi_{1}^{\prime}\left(t_{n}\right), \quad n \rightarrow \infty
$$


and in view of (24) we have

$$
t_{n} \xi_{n}=O\left(\Phi_{2}\left(\varphi_{1}\left(t_{n}\right)\right)\right), \quad n \rightarrow \infty
$$

whence

$$
\xi_{n}\left(t_{n}-\Phi_{1}^{\prime}\left(\kappa_{n}\left(\Phi_{1}\right)\right)\right)=\xi_{n}\left(t_{n}-\Phi_{1}^{\prime}\left(\varphi\left((1+o(1)) t_{n}\right)\right)\right)=o\left(\xi_{n} t_{n}\right)=o\left(\Phi_{2}\left(\varphi_{1}\left(t_{n}\right)\right)\right)
$$

as $n \rightarrow \infty$. Finally, (27) implies

$$
\begin{aligned}
\frac{\xi_{n} \Phi_{2}^{\prime}\left(\kappa_{n}\left(\Phi_{1}\right)\right)}{\Phi_{2}\left(\kappa_{n}\left(\Phi_{1}\right)\right)}= & \frac{\xi_{n} \Phi_{2}^{\prime}\left(\varphi_{1}(1+o(1)) t_{n}\right)}{\Phi_{2}\left(\varphi_{1}(1+o(1)) t_{n}\right)}=o\left(\frac{\xi_{n} \Phi_{1}^{\prime}\left(\varphi_{1}(1+o(1)) t_{n}\right)}{\Phi_{2}\left(\varphi_{1}(1+o(1)) t_{n}\right)}\right)= \\
& =o\left(\frac{\xi_{n} t_{n}}{\Phi_{2}\left(\varphi_{1}\left(t_{n}\right)\right)}\right)=o(1), n \rightarrow \infty
\end{aligned}
$$

The asymptotic equality (21) follows from (25) and (27)-(29).

Lemma 6. Let $\Phi_{1} \in \Omega^{*}(A), \varphi_{1}^{\prime} \in L^{0}$ and $\Phi_{2}$ be weakly subordinated to $\Phi_{1}$. Suppose that $\Phi_{2}^{\prime}(\sigma)=o\left(\sigma \Phi_{1}^{\prime \prime}(\sigma)\right)$ as $\sigma \uparrow A$, the conditions (3) as $\sigma \uparrow A$ hold and

$$
\left.\xi_{n} \Phi_{1}^{\prime}\left(\kappa_{n}\left(\Phi_{1}\right)\right)\right)=o\left(G_{2}\left(t_{n}, t_{n+1}, \Phi_{1}\right)\right), \quad \Phi_{2}\left(\kappa_{n}\left(\Phi_{1}\right)\right)=o\left(G_{2}\left(t_{n}, t_{n+1}, \Phi_{1}\right)\right)
$$

as $n \rightarrow \infty$. Suppose also that

$$
\Phi_{*}(\sigma)=\Phi_{1}(\sigma)+(\tau-\varepsilon) \Phi_{2}(\sigma), \quad \Phi^{*}(\sigma)=\Phi_{1}(\sigma)+(\tau+\varepsilon) \Phi_{2}(\sigma)
$$

where $\varepsilon \in(0,|\tau|)$. Then the inequality $G_{1}\left(t_{n}, t_{n+1}, \Phi^{*}\right) \geq \Phi_{*}\left(\varkappa_{n}\left(\Phi^{*}\right)\right)$ for some sequence $\left(t_{n}\right) \uparrow+\infty$ implies the inequality

$$
0<G_{2}\left(t_{n}, t_{n+1}, \Phi_{1}\right)-G_{1}\left(t_{n}, t_{n+1}, \Phi_{1}\right)=2 \varepsilon\left(1+o(1) \Phi_{2}\left(\varphi_{1}\left(t_{n}\right)\right), \quad n \rightarrow \infty .\right.
$$

Proof. The inequality $G_{1}\left(t_{n}, t_{n+1}, \Phi^{*}\right) \geq \Phi_{*}\left(\varkappa_{n}\left(\Phi^{*}\right)\right)$ is equivalent to the inequality

$$
0<G_{2}\left(t_{n}, t_{n+1}, \Phi^{*}\right)-G_{1}\left(t_{n}, t_{n+1}, \Phi^{*}\right) \leq 2 \varepsilon \Phi_{2}\left(\varkappa_{n}\left(\Phi^{*}\right)\right) .
$$

Using (22) with $\Phi^{*}$ and $\tau+\varepsilon$ instead $\Phi$ and $\tau$ we have

$$
\begin{gathered}
G_{2}\left(t_{n}, t_{n+1}, \Phi^{*}\right)=G_{2}\left(t_{n}, t_{n+1}, \Phi_{1}\right)+(\tau+\varepsilon) \Phi_{2}\left(\kappa_{n}\left(\Phi_{1}\right)\right)- \\
-(1+o(1))(\tau+\varepsilon) \xi_{n} \Phi_{1}^{\prime}\left(\kappa_{n}\left(\Phi_{1}\right)\right)-(1+o(1))(\tau+\varepsilon) \xi_{n} \Phi_{2}^{\prime}\left(\kappa_{n}\left(\Phi_{1}\right)\right), \quad n \rightarrow \infty .
\end{gathered}
$$

On the other hand, (23) implies

$G_{1}\left(t_{n}, t_{n+1}, \Phi^{*}\right)=G_{1}\left(t_{n}, t_{n+1}, \Phi_{1}\right)-(1+o(1))(\tau+\varepsilon) \xi_{n} t_{n}+(1+o(1))(\tau+\varepsilon) \Phi_{2}\left(\varphi_{1}\left(t_{n}\right)\right), \quad n \rightarrow \infty$.

Therefore, from (32) we obtain

$$
\begin{gathered}
0<G_{2}\left(t_{n}, t_{n+1}, \Phi_{1}\right)-G_{1}\left(t_{n}, t_{n+1}, \Phi_{1}\right)+(\tau+\varepsilon) \Phi_{2}\left(\kappa_{n}\left(\Phi_{1}\right)\right)- \\
-(1+o(1))(\tau+\varepsilon) \xi_{n} \Phi_{1}^{\prime}\left(\kappa_{n}\left(\Phi_{1}\right)\right)-(1+o(1))(\tau+\varepsilon) \xi_{n} \Phi_{2}^{\prime}\left(\kappa_{n}\left(\Phi_{1}\right)\right)- \\
+(1+o(1))(\tau+\varepsilon) \xi_{n} t_{n}-(1+o(1))(\tau+\varepsilon) \Phi_{2}\left(\varphi_{1}\left(t_{n}\right)\right) \leq 2 \varepsilon \Phi_{2}\left(\varkappa_{n}\left(\Phi^{*}\right)\right) .
\end{gathered}
$$


Since $t_{n}=\Phi^{\prime}\left(\varphi\left(t_{n}\right)\right) \leq \Phi^{\prime}\left(\varkappa_{n}\left(\Phi_{1}\right)\right)$, from (30) it follows that

$$
\frac{t_{n} \xi_{n}}{G_{2}\left(t_{n}, t_{n+1}, \Phi_{1}\right)} \rightarrow 0, \frac{\Phi_{2}\left(\varkappa_{n}\left(\Phi_{1}\right)\right)}{G_{2}\left(t_{n}, t_{n+1}, \Phi_{1}\right)} \rightarrow 0, \quad n \rightarrow \infty .
$$

Therefore, (33) implies (14) and, thus, (5). Using (5) and the equality $\Phi_{2}\left(\kappa_{n}\left(\Phi_{1}\right)\right)=(1+$ $o(1)) \Phi_{2}\left(\varphi_{1}\left(t_{n}\right)\right)$ as $n \rightarrow \infty$, as in the proof of Lemma 5 , from (33) we have

$$
\begin{gathered}
G_{2}\left(t_{n}, t_{n+1}, \Phi_{1}\right)-G_{1}\left(t_{n}, t_{n+1}, \Phi_{1}\right) \leq(1+o(1))(\tau+\varepsilon) \xi_{n} \Phi_{1}^{\prime}\left(\kappa_{n}\left(\Phi_{1}\right)\right)+ \\
+(1+o(1))(\tau+\varepsilon) \xi_{n} \Phi_{2}^{\prime}\left(\kappa_{n}\left(\Phi_{1}\right)\right)-(1+o(1))(\tau+\varepsilon) \xi_{n} t_{n}+ \\
+o\left(\Phi_{2}\left(\varphi_{1}\left(t_{n}\right)\right)\right)+2 \varepsilon \Phi_{2}\left(\varkappa_{n}\left(\Phi_{1}\right)\right), \quad n \rightarrow \infty .
\end{gathered}
$$

whence, repeating the proof of Lemma 5, we obtain (31).

4. Main results. From Lemmas 1 and 4 the following generalization of Theorem A follows.

Theorem 1. Let $\tau \in \mathbb{R} \backslash\{0\}$ and either $A=0$ or $A=+\infty$. Suppose that $\Phi_{1} \in \Omega(A)$, $\varphi_{1}^{\prime} \in L^{0}$ and $\Phi_{2}$ is weakly subordinated to $\Phi_{1}$. Then the asymptotic inequality (1) holds with $\sigma \uparrow A$ instead $\sigma \rightarrow+\infty$ if and only if the asymptotic inequality (2) holds.

Indeed, for $\Phi(\sigma)=\Phi_{1}(\sigma)+(\tau+\varepsilon) \Phi_{2}(\sigma)\left(\sigma \in\left[\sigma_{0}(\tau+\varepsilon), A\right)\right.$, where $\varepsilon \in(0,|\tau|)$ is an arbitrary number, by Lemma 4 we have

$$
t \Psi(\varphi(t))=t \Psi_{1}\left(\varphi_{1}(t)\right)-(\tau+\varepsilon)(1+o(1)) \Phi_{2}^{\prime}\left(\varphi_{1}(t)\right), \quad t \rightarrow+\infty .
$$

Then, Lemma 1 completes the proof of Theorem 1.

Theorem 2. Let $\tau \in \mathbb{R} \backslash\{0\}$ and either $A=0$ or $A=+\infty$. Suppose that $\Phi_{1} \in \Omega(A)$, $\varphi_{1}^{\prime} \in L^{0}, \Phi_{2}$ is weakly subordinated to $\Phi_{1}, \Phi_{2}^{\prime}(\sigma)=o\left(\sigma \Phi_{1}^{\prime \prime}(\sigma)\right)$ as $\sigma \uparrow A$ and conditions (3) as $\sigma \uparrow A$ hold. If the asymptotic equality (4) holds for some increasing to $+\infty$ sequence $\left(t_{n}\right)$, satisfying (5) and (6), then the asymptotic equality (7) holds as $\sigma \uparrow A$.

Proof. By the assumptions for an arbitrary $\varepsilon \in(0,|\tau|)$ the inequality

$$
P\left(t_{n}\right) \geq-t_{n} \Psi_{1}\left(\varphi_{1}\left(t_{n}\right)\right)+(\tau-\varepsilon) \Phi_{2}\left(\varphi_{1}\left(t_{n}\right)\right)
$$

holds for a some increasing to $+\infty$ sequence $\left(t_{n}\right)$, satisfying (5) and (6). By Lemma 5 for the function $\Phi(\sigma)=\Phi_{1}(\sigma)+(\tau-\varepsilon) \Phi_{2}(\sigma)\left(\sigma \in\left[\sigma_{0}(\tau-\varepsilon), A\right)\right)$ we have (21). But in view of (16) and (3)

$$
\frac{\Phi_{2}^{\prime}(\varphi(t))}{\Phi_{2}\left(\varphi_{1}(t)\right)}=\frac{1}{\Phi_{2}^{\prime}\left(\varphi_{1}(t)\right)} \Phi_{2}\left(\varphi(t)-(1+o(1)) \frac{\Phi_{2}^{\prime}\left(\varphi_{1}(t)\right)}{\Phi_{2}^{\prime \prime}\left(\varphi_{1}(t)\right)}\right) \rightarrow 1, \quad t \rightarrow+\infty .
$$

Therefore, (21) and (6) implies $G_{2}\left(t_{n}, t_{n+1}, \Phi\right)-G_{1}\left(t_{n}, t_{n+1}, \Phi\right)=o\left(\Phi_{2}\left(\varphi\left(t_{n}\right)\right)\right), n \rightarrow \infty$, and by Lemma 2 for all $n \geq n_{0}$ and all $\sigma \in\left[\varphi\left(t_{n}\right), \varphi\left(t_{n+1}\right)\right]$

$$
\begin{gathered}
Q(\sigma) \geq \Phi(\sigma)+G_{1}\left(t_{n}, t_{n+1}, \Phi\right)-G_{2}\left(t_{n}, t_{n+1}, \Phi\right)=\Phi(\sigma)+o\left(\Phi_{2}\left(\varphi\left(t_{n}\right)\right)\right)= \\
=\Phi(\sigma)+o\left(\Phi_{2}(\sigma)\right)=\Phi_{1}(\sigma)+(\tau-\varepsilon+o(1)) \Phi_{2}(\sigma), \quad \sigma \uparrow A .
\end{gathered}
$$

In view of the arbitrariness of $\varepsilon$ Theorem 2 is proved.

Finally, we prove the following main theorem. 
Theorem 3. Let $\tau \in \mathbb{R} \backslash\{0\}$ and either $A=0$ or $A=+\infty$. Suppose that $\Phi_{1} \in \Omega^{*}(A)$, $\varphi_{1}^{\prime} \in L^{0}, \Phi_{2}$ is weakly subordinated to $\Phi_{1}, \Phi_{2}^{\prime}(\sigma)=o\left(\sigma \Phi_{1}^{\prime \prime}(\sigma)\right)$ as $\sigma \uparrow A$ and the conditions (3) as $\sigma \uparrow A$ hold.

Suppose also that either $\sigma \Phi_{1}^{\prime}(\sigma)=O\left(\Phi_{1}(\sigma)\right)$ as $\sigma \uparrow A$ (when $A=0$ or $A=+\infty$ ), or $\Phi_{2}^{\prime}(\sigma)=o\left(\Phi_{1}^{\prime \prime}(\sigma)\right)$ and $\Phi_{1}^{\prime}(\sigma)=O\left(\Phi_{1}(\sigma)\right)$ as $\sigma \uparrow A$ when $A=+\infty$.

In order that

$$
Q(\sigma)=\Phi_{1}(\sigma)+(1+o(1)) \tau \Phi_{1}(\sigma), \quad \sigma \uparrow A,
$$

it is necessary and sufficient that for every $\varepsilon>0$ : 1) for all $t \geq t_{0}=t_{0}(\varepsilon)$

$$
P(t) \leq-t \Psi_{1}\left(\varphi_{1}(t)\right)+(\tau+\varepsilon) \Phi_{2}\left(\varphi_{1}(t)\right)
$$

2) there exists an increasing to $+\infty$ sequence $\left(t_{n}\right)$ such that

$$
\left.P\left(t_{n}\right) \geq-t_{n} \Psi_{1}\left(\varphi_{1}\left(t_{n}\right)\right)+(\tau-\varepsilon)\right) \Phi_{2}\left(\varphi_{1}\left(t_{n}\right)\right), \quad n \rightarrow+\infty
$$

and

$$
\lim _{n \rightarrow \infty} \frac{G_{2}\left(t_{n}, t_{n+1}, \Phi_{1}\right)-G_{1}\left(t_{n}, t_{n+1}, \Phi_{1}\right)}{\Phi_{2}\left(\varphi_{1}\left(t_{n}\right)\right)}=0
$$

Proof. If (34) holds then

$$
\Phi_{*}(\sigma)=\Phi_{1}(\sigma)+(\tau-\varepsilon) \Phi_{2}(\sigma) \leq Q(\sigma) \leq \Phi^{*}(\sigma)=\Phi_{1}(\sigma)+(\tau+\varepsilon) \Phi_{2}(\sigma)
$$

for arbitrary $\varepsilon \in(0,|\tau|)$ and all $\sigma \in[\sigma(\varepsilon), A)$. Hence by Lemmas 1 and 4 we get

$$
P(t) \leq-t \Psi_{1}\left(\varphi_{1}(t)\right)+(\tau+\varepsilon)(1+o(1)) \Phi_{2}\left(\varphi_{1}(t)\right)
$$

as $t \rightarrow+\infty$, i. e. (35) holds.

By Lemma 3 there exists an increasing to $+\infty$ sequence $\left(t_{n}\right)$ of positive numbers such that $P\left(t_{n}\right) \geq-t_{n} \Psi_{*}\left(\varphi_{*}\left(t_{n}\right)\right)$ and $G_{1}\left(t_{n}, t_{n+1}, \Phi^{*}\right) \geq \Phi_{1}\left(\varkappa_{n}\left(\Phi^{*}\right)\right)$. From the inequality $P\left(t_{n}\right) \geq$ $-t_{n} \Psi_{*}\left(\varphi_{*}\left(t_{n}\right)\right)$ in view of Lemma 4 we have $(36)$.

Further, since the condition $\sigma \Phi_{1}^{\prime}(\sigma)=O\left(\Phi_{1}(\sigma)\right)$ as $\sigma \uparrow A$ (when $A=0$ or $A=+\infty$ ), implies (30) (see the proof of Proposition 1), by Lemma 6 the relation (31) holds, whence in view of arbitrariness of $\varepsilon$ we obtain (37). If $A=+\infty$ then the condition $\Phi_{2}^{\prime}(\sigma)=o\left(\Phi_{1}^{\prime \prime}(\sigma)\right)$ and $\Phi_{1}^{\prime}(\sigma)=O\left(\Phi_{1}(\sigma)\right)$ as $\sigma \rightarrow+\infty$ imply (30) (see the proof of Proposition 2) and, therefore, we obtain again (31) and, thus, (37).

Now we will prove the sufficity. By Lemmas 1 and 4 in view of the arbitrariness of $\varepsilon$ the condition (35) implies the inequality $Q(\sigma) \leq \Phi_{1}(\sigma)+(\tau+\varepsilon) \Phi_{2}(\sigma)$. On the other hand, by Lemmas 2 and 4 from (36) we have $Q(\sigma) \geq \Phi(\sigma)+G_{1}\left(t_{n}, t_{n+1}, \Phi\right)-G_{2}\left(t_{n}, t_{n+1}, \Phi\right)$ for all $n \geq n_{0}$ and all $\sigma \in\left[\varphi\left(t_{n}\right), \varphi\left(t_{n+1}\right)\right]$, where $\Phi(\sigma)=\Phi_{1}(\sigma)+(\tau-\varepsilon) \Phi_{2}(\sigma)$. Since $\Phi_{1} \in \Omega^{*}(A)$, from (37) we obtain (5). Therefore, by Lemma 5 for all $\sigma \in\left[\varphi\left(t_{n}\right), \varphi\left(t_{n+1}\right)\right]$ as $n \rightarrow \infty$

$$
Q(\sigma) \geq \Phi_{1}(\sigma)+(\tau-\varepsilon) \Phi_{2}(\sigma)+G_{1}\left(t_{n}, t_{n+1}, \Phi_{1}\right)-G_{2}\left(t_{n}, t_{n+1}, \Phi_{1}\right)+o\left(\Phi_{2}\left(\varphi\left(t_{n}\right)\right)\right),
$$

whence as in the proof of Theorem 2 we obtain the inequality $Q(\sigma) \geq \Phi_{1}(\sigma)+(\tau-\varepsilon+$ $o(1)) \Phi_{2}(\sigma)$ as $\sigma \uparrow A$. In view of the arbitrariness of $\varepsilon$ the proof of Theorem 3 is complete. 


\section{REFERENCES}

1. Sheremeta M.M. Asymptotical behaviour of Laplace-Stiltjes integrals, Lviv: VNTL Publishers, 2010. $211 \mathrm{p}$.

2. Sheremeta M.M., Fedynyak S.I. On the derivative of a Dirichlet series// Sibirsk. Mat. Zh. - 1998. - V.39, №1. - P. 206-223.

3. Sheremeta M.M., Sumyk O.M. Connection between the growth of Young conjugated functions// Mat. Stud. - 1999. - V.11, №1. - P. 41-47.

4. Zabolotskyi M.V., Sheremeta M.M. Generalization of the Lindelöf theorem// Ukr. mat. journ. - 1998. V.50, №9 - P. 1177-1192. (in Ukrainian)

5. Sumyk O.M. Asymptotic behaviour of Young conjugated functions and application to the Dirichlet series, Candid. diss., Lviv, 2002. - 150 p.

6. Sheremeta M.M. Estimates of the maximal term of entire Dirichlet series in terms of two-member asymptotics// Mat. Stud. - 2000. - V.14, №2. - P. 159-164.

7. Sheremeta M.M. Two-member asymptotics of Young conjugated functions and problems of behaviour of positive sequences// Mat. Stud. - 2000. - V.14, №2. - P. 217-220.

Ivan Franko National University of Lviv

m_m_sheremeta@list.ru

Received 5.07.2016 